\title{
Drugging the schistosome zinc-dependent HDACs: current progress and future perspectives
}

Schistosomes, like many eukaryotic pathogens, typically display morphologically distinct stages during their life cycles. Epigenetic mechanisms underlie the pathogens' morphological transformations, and the targeting of epigenetics-driven cellular programs therefore represents an Achilles' heel of parasites. To speed up the search for new antiparasitic agents, drugs validated for other diseases can be rationally optimized into antiparasitic therapeutics. Specifically, zinc-dependent histone deacetylases (HDACs) are the most explored targets for epigenetic therapies, notably for anticancer treatments. This review focuses on the development of drug-leads inhibiting HDACs from schistosomes. More precisely, current progress on Schistosoma mansoni HDAC8 (smHDAC8) provided a proof of concept that targeting epigenetic enzymes is a valid approach to treat diseases caused by schistosomes, and possibly other eukaryotic pathogens.

\section{Schistosomiasis: key facts}

Schistosomiasis, or bilharzia, is a parasitic disease caused by trematode flatworms of the genus Schistosoma (S. mansoni, S. japonicum and $S$. haematobium are the main species of medical relevance) [1,2]. Larval forms of the parasites, which are released by intermediate hosts, specific freshwater snail species, penetrate the skin of people in water. In the body, the larvae develop into adult schistosomes, which live in the blood vessels (Figure 1). The females release eggs, some of which are passed out of the body in the urine or feces. Others are trapped in body tissues, triggering an immune reaction that causes the pathological symptoms associated with schistosomiasis. In urinary schistosomiasis, there is progressive damage to the bladder, urethra and kidneys [3]. In intestinal schistosomiasis, there is progressive enlargement of the liver and spleen, intestinal damage, and hypertension of the abdominal blood vessels [4].

According to WHO statistics, schistosomes infect around 230 million people worldwide and cause at least 300,000 deaths yearly, with about 800 million people further at risk of infection [5]. The dependence of the control of schistosomiasis on mass treatment with a single drug, praziquantel (Figure 2) [6], and the consequent risk of the appearance of resistant strains raise the specter of widespread drug resistance/tolerance. Indeed, praziquantelresistant schistosome strains have already been reported [7-9], and these findings rendered the search for new antischistosomal drugs a strategic priority.

\section{Epigenetic networks: an Achilles' heel of eukaryotic parasites}

All cells in any organism inherit the identical genetic material. The ability of cells to maintain the unique properties and biological functions of specific tissues and organs, but also to adapt to changing environmental cues, is in large part due to differences in the packaging of this genetic material. These differences direct distinct cellular gene expression programs without involving changes in the original DNA sequence of the organism $[10,11]$.

The packaged genetic material in the cell nucleus is termed chromatin. The
Martin Marek', Guilherme Oliveira², Raymond J Pierce ${ }^{3}$, Manfred Jung ${ }^{4}$, Wolfgang Sippl ${ }^{5}$ \& Christophe Romier ${ }^{*}, 1$ 'Département de Biologie Structurale Intégrative, Institut de Génétique et Biologie Moléculaire et Cellulaire (IGBMC), Université de Strasbourg (UDS), CNRS, INSERM, 1, rue Laurent Fries, 67404 Illkirch Cedex, France

${ }^{2}$ Genomics \& Computational Biology Group, Centro de Pesquisas René Rachou, Fundaçāo Oswaldo Cruz, Belo Horizonte, MG-30190-002, Minas Gerais, Brazi

${ }^{3}$ Center for Infection \& Immunity of Lille, INSERM U1019-CNRS UMR 8204 , Université de Lille, Institut Pasteur de Lille, 1 rue Professeur Calmette, F-59019 Lille Cedex, France

${ }^{4}$ Institute of Pharmaceutical Sciences, Albert-Ludwigs-Universität Freiburg, Albertstraße 25, 79104 Freiburg. Germany

${ }^{5}$ Institute of Pharmacy, Martin-LutherUniversität Halle-Wittenberg, WolfgangLangenbeck-Straße 4, 06120 Halle/Saale, Germany

*Author for correspondence:

Tel.: (+33) 369485294 Fax: (+33) 388653201 romier@igbmc.fr 


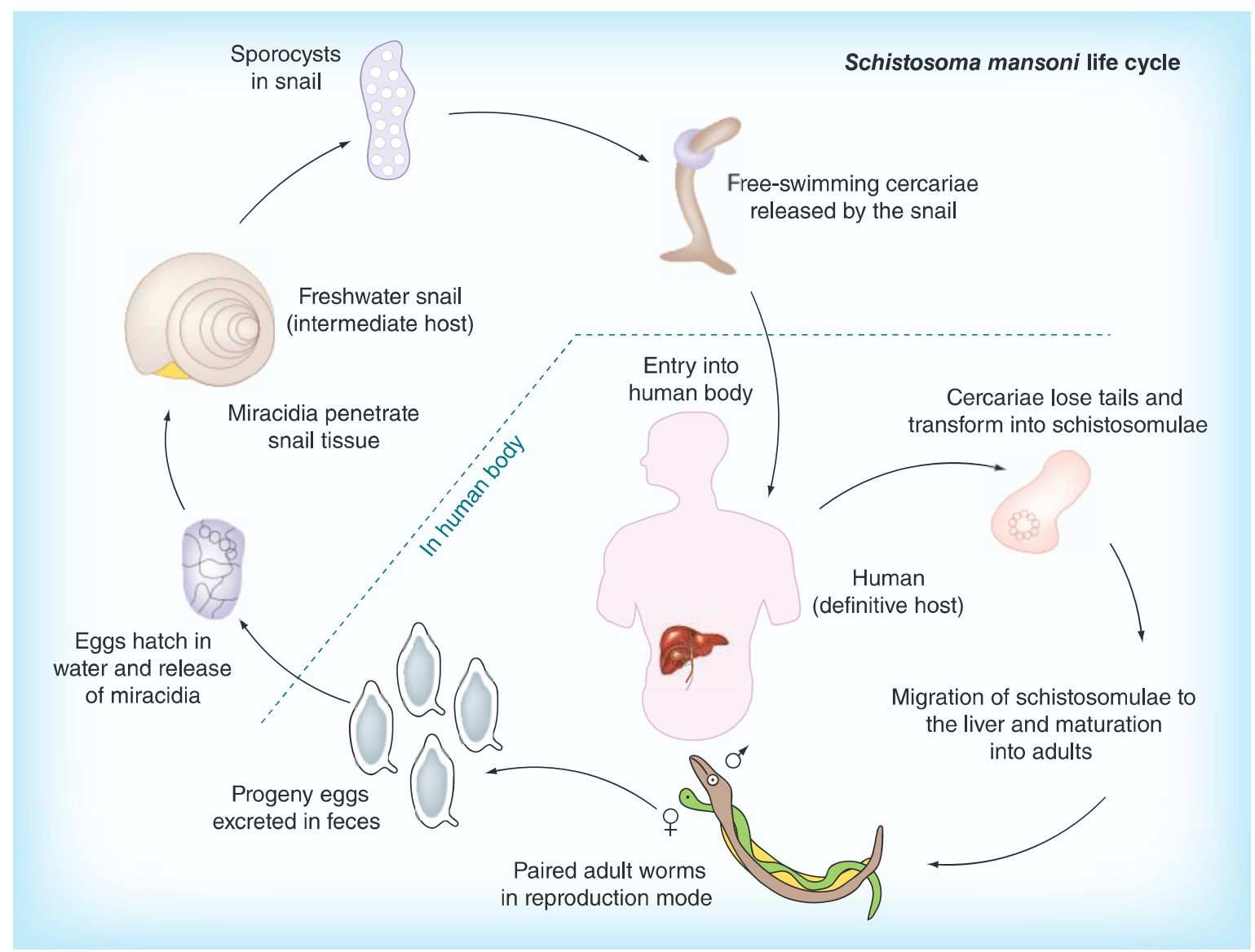

Figure 1. Life cycle of the blood fluke Schistosoma mansoni. Progeny eggs are eliminated from infected human host with feces into water, where they hatch and release miracidia. The miracidia swim and penetrate the intermediate host, specific freshwater snail species. Within the snail, the miracidia progress through two generations of sporocysts to generate cercariae. The free-swimming cercariae are released from the snail into water, where they search for and penetrate the skin of the definitive human host. During penetration, the cercariae lose their forked tail and transform into schistosomulae. The schistosomulae migrate through the vascular system to the liver, where they mature into adults. The paired adult worms (male and female) travel to intestinal veins in the bowel, where the female start to lay eggs. The progeny eggs are eliminated with feces into water, which completes the life cycle.

elementary building block of chromatin is the nucleosome. It is composed of a core histone octamer composed of two copies of each of four histone molecules $(\mathrm{H} 2 \mathrm{~A}, \mathrm{H} 2 \mathrm{~B}, \mathrm{H} 3$ and $\mathrm{H} 4)$ around which is wrapped 147 bp base pairs of DNA [12]. Packing of nucleosomes against each other leads to higher order chromatin structure that, depending on its arrangement, favors or represses access to the underlying genetic material and thus regulates other nuclear processes [13].

Specific epigenetic mechanisms modulate chromatin, altering its higher order structure and impacting on nuclear functions. The term 'epigenetics' describes chromatin-based events that regulate DNA-based processes [14]. Several types of epigenetic mechanisms have been characterized that collaborate to modulate chromatin structure. These range from the reversible covalent modifications of histones and DNA bases to the use of histone variants, ATP-dependent nucleosome remodeling and noncoding RNA-mediated mechanisms $[15,16]$.

Over recent decades, enormous research efforts have been dedicated to the study of covalent histone modifications $[15,17-19]$ that are carried out by chromatin-modifying enzymes in a precisely regulated manner. The term 'epigenome' describes a record of the chemical changes to the chromatin and chromatin-associated macromolecules of an organism. Changes to the epigenome can result in changes to the structure of chromatin and changes to the function of the genome [20]. Consequently, perturbations in these highly coordinated epigenetic events may have profound biological implications with the induction of various pathologies, such as cancer [21-23].

Human parasitic diseases are caused by numerous taxonomically diverse infectious eukaryotic 
(A)<smiles>O=C(C1CCCCC1)N1CC(=O)N2CCc3ccccc3C2C1</smiles>

(B)<smiles>CCCC(CCC)C(=O)O</smiles>

(E)

(D)<smiles>O=C(NO)c1sc2ccccc2c1Cl</smiles>

F)<smiles>CN(C)c1ccc(C(=O)NCCCCCCC(=O)NO)cc1</smiles>

(H)<smiles>O=C(CCCCCCC(=O)Nc1ccccc1)NO</smiles>

(J)<smiles>CS(=O)(=O)OCCCCCC(=O)Nc1ccccc1</smiles>

(C)

Romidepsin

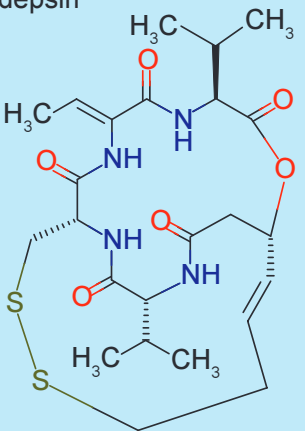

(G)
(1)<smiles>O=C(/C=C/c1cccc(S(=O)(=O)Nc2ccccc2)c1)NO</smiles>

(K)

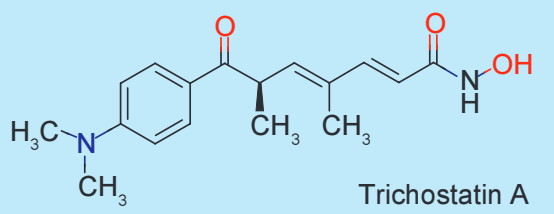

Figure 2. Structures of inhibitors.(A) Praziquantel, (B) valproic acid, (C) Romidepsin, (D) J1075, (E) J1038, (F) M344, (G) PCl-34051, (H) SAHA, (I) Belinostat, (J) DS45 and (K) Trichostatin A.

organisms. Some eukaryotic parasites require adaptation to different environments such as specific vectors and definitive host species ranging from invertebrates, vertebrates or plants to the external environment, such as water, soil, etc. Crucially, most human eukaryotic pathogens typically transform into several morphologically distinct stages during their life cycles, where epigenetic mechanisms are supposed to play essential roles in physiological adaptations [24,25]. For example, it has recently been shown that histone deacetylase 2 (HDAC2), an essential epigenetic actor in the malaria parasite Plasmodium falciparum, is a global silencer of virulence gene expression and controls the frequency of switching from the asexual cycle to sexual development [26]. But many other examples of epigenetic plasticity in host-pathogen interactions have been described [24]. Recently reported research provided strong experimental evidence that the targeting of key epigenetic players represents the Achilles' heel of the eukaryotic pathogens, which opens up new avenues in the drugging parasites' epigenomes and treatment of human and animal parasitic infections [27-31].

\section{Reversible protein acetylation is an essential process of epigenetic pathways}

Post-translational covalent modifications of histones influence the conformation of chromatin by changing interactions between the histones and the nucleosomal DNA, and by recruiting epigenetic complexes that will alter nucleosome composition (e.g., replacement of canonical histone by histone variants) and positioning (e.g., ATP-dependent chromosome remodeling) [11,15,17-18]. Another class of modification concerns the covalent attachment of relatively small chemical groups to specific amino acids, as well as covalent attachment of small proteins, for example, ubiquitin and SUMO (Small Ubiquitin-like MOdifier). Lysine/

\section{Key term}

Drugging parasites' epigenomes: Epigenetic plasticity directs the parasites' morphogenetic and developmental programs. These facts, coupled with recent progress in showing that enzymes involved in epigenetic regulations can be selectively controlled by small-molecule drugs, have boosted interest in exploiting the therapeutic potential of epigenetic targets. 
arginine methylation, serine/threonine/tyrosine phosphorylation and lysine acetylation are historically the most investigated chemical reactions, although recent discoveries showed that additional covalent modifications, among others for example crotonylation, succinylation and malonylation, also frequently occur $[32,33]$. The enzymes involved in placement of these groups on histones are referred to as writers and the enzymes involved in the specific removal of these chemical groups are referred to as erasers. Proteins recognizing and binding to these specific epigenetic marks on histones are known as readers (e.g., bromodomain-, chromodomain- and tudor domain-containing proteins) [34,35] (see Figure 3 for a schematic overview of these covalent modifications of histones).

Reversible acetylation of $\varepsilon$-amino groups of lysine residues on cellular proteins is currently considered to be an ancient post-translational modification of proteins, which is conserved from prokaryotes to mammals [33]. Acetylations of histone lysines residues are catalyzed by histone acetyltransferases (HATs), a diverse family of enzymes that utilize acetyl-CoA as a common acetyl group donor [36]. Protein acetylation results in charge neutralization of the lysine side chain, hence, changing the mutual electrostatic interactions with the negatively charged DNA and neighboring nucleosomes [37]. It is widely accepted that histone acetylation triggers a switch from condensed heterochromatin to more open, uncondensed euchromatin, which is, for example, a prerequisite for an exposure of gene promoter sequences to transcriptional machinery [11,17-18]. In addition, site-specific lysine acetylation also forms a docking platform for the recruitment of transcriptional complexes sensing 'transcription permissive' chromatin via protein components specifically binding to acetylated lysine residues, such as bromodomain-containing proteins $[38,39]$.

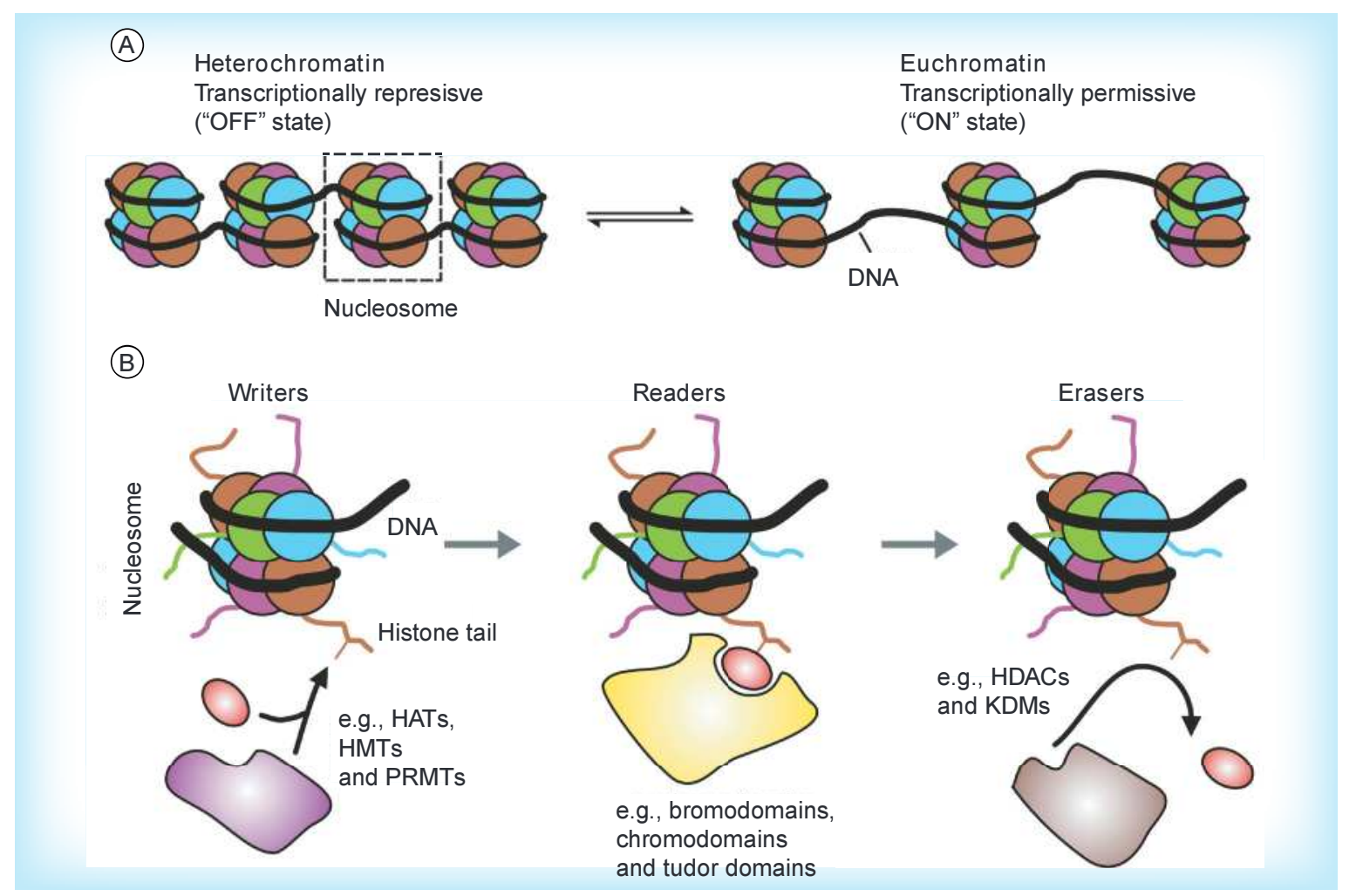

Figure 3. Cartoon of the conformational transition of chromatin structure triggered by covalent modifications of histones. (A) The conformational transition of chromatin from a condensed, transcriptionally repressive heterochromatin to an open, transcriptionally permissive euchromatin. (B) Overview of reversible chromatin modifications. Epigenetic writers such as HATs, HMTs, PRMTs and kinases catalyze the attachment of epigenetic marks (e.g., acetyl, methyl and phosphate chemical groups) on amino acid residues on histone tails. Epigenetic readers such as proteins containing bromodomains, chromodomains and tudor domains recognize and bind to these epigenetic marks. Epigenetic erasers such as HDACs, KDMs and phosphatases catalyze the removal of epigenetic marks. As a consequence, histone modifications alter chromatin structure, which facilitates recruitments of a variety of macromolecular complexes involved in various DNA-templated processes, including gene transcription, DNA replication and DNA repair.

HAT: Histone acetyltransferase; HDAC: Histone deacetylase; HMT: Histone methyltransferase; KDM: Lysine demethylase; PRMT: protein arginine methyltransferase. 
Site-specific deacetylation of lysine residues in cellular proteins is catalyzed by HDACs. Deacetylated histones are often associated with gene repression, as the removal of acetyl groups restores the basic charge of lysine residues that can interact tightly with nucleosomal DNA and neighboring nucleosomes via electrostatic interactions. Moreover, the acetylerased histones are generally not recognized by the bromodomain-containing transcriptional complexes.

Thus far, 18 mammalian HDACs have been identified that were extensively studied over recent decades. HDACs are divided into four classes based on sequence homology and phylogenetic relationships [40]. Class $\mathrm{I}$ is similar to the yeast $\mathrm{Rpd} 3$ and Hos 1/2 enzymes, and contains the enzymes HDAC $1,2,3$ and 8 [41]. Class II is related to the yeast Hos3 and HDA1 enzymes, and this class is further subdivided into subclass IIa (HDAC 4, 5, 7 and 9) and subclass IIb (HDAC6 and HDAC10). Class III is made up of the sirtuins $1-7$, and this group of enzyme is similar to yeast Sir2. Finally, class IV contains a single enzyme, HDAC 11. Members of classes I, II and IV represent so-called 'classical' HDACs (hereafter termed 'HDACs') utilizing zinc to activate a coordinated water molecule, which together with active-site histidine and aspartate side chains mediate nucleophilic catalysis on the acetylated lysine substrate $[35,42-43]$. Unlike classical HDACs, sirtuins represent a distinct group of deacetylases that use $\mathrm{NAD}^{+}$as a cofactor for catalysis [44].

Importantly, reversible protein acetylation occurs not only on histones, but also on a large number of non-histone proteins, affecting their physico-chemical properties, and thereby regulating their functions in many different biological processes [17,18]. In consequence, imbalance of protein acetylation is a common aspect of many disorders, such as cancer, neurodegenerative diseases and metabolic disorders [45,46]. Finally, the enzymes involved in the placement of acetyl groups on histones and the enzymes involved in the specific removal of this chemical group, protein acetylation erasers, are so far the most studied epigenetic actors in schistosomes [27-28,47-50]. The diversity and biological functions of proteins recognizing other epigenetic marks on histones or nucleotides such as bromodomain, chromodomain and tudor domain-containing proteins [51], have not yet been investigated in schistosomes.

This review focuses on the current knowledge and perspectives in drugging schistosome zinc-dependent HDACs. Notably, it builds on recent structural, biochemical and in vivo work that revealed unexpected pharmacological opportunities for the development of structure-based drugs for antiparasitic epigenetic therapy.

\section{Key term}

Antiparasitic epigenetic therapy: The use of drugs and other epigenome-influencing approaches dedicated to cure diseases caused by eukaryotic parasites. The objective of this strategy is to maximize the selectivity of the used drugs in order to avoid possible side effects.

\section{Repertoire of protein acetylation erasers in schistosomes}

Schistosomes are eukaryotic, multicellular, vertebrate-infecting flatworms, which extensively utilize epigenetic tools and mechanisms to govern their complex parasitic lifestyle [48,50,52]. Interestingly, it has recently been demonstrated that the reversible protein acetylation mediated by HATs and HDACs is an essential process of these epigenetic regulations that drive the developmental program of schistosomes [27-28,50].

Enzymes erasing acetyl group from lysine residues, HDACs, are thus far the most explored schistosome epigenetic tools $[27,47,49]$. Bioinformatic searches identified six 'classical' zinc-utilizing HDACs encoded in the $S$. mansoni genome (Figure 4) [47,48]. Three of them are orthologs of class I HDACs, namely, HDAC1, 3 and 8. The $S$. mansoni HDAC1 (smHDAC1) and $S$. mansoni HDAC3 (smHDAC3) are highly conserved compared with their human counterparts, whilst $S$. mansoni HDAC8 (smHDAC8) is more distant and contains several relatively large insertions in the protein sequence (Figure 4). No HDAC2 ortholog was identified in schistosome genomes, which is in agreement with the fact that this isoform seems to be the outcome of a vertebrate-specific duplication of the HDAC1 gene [53]. The remaining 'classical' schistosome HDACs called smHDAC4, 5 and 6, as inferred from their sequence similarities to mammalian orthologs [48,50], represent class II HDACs, as shown in Figure 4 . These class II HDACs have not been functionally characterized yet. Nevertheless, sequence comparisons revealed that smHDAC4 and smHDAC5 are distinguished from their human counterparts based on the presence of a catalytically essential tyrosine residue normally found only in schistosome class I and class IIb HDACs. This tyrosine is replaced by a histidine residue in vertebrate class IIa HDACs (Figure 4). Because of this change, vertebrate class IIa HDACs exhibit basal enzymatic activities on acetyl-lysines, and Lahm and coworkers [54] proposed that these enzymes may process restricted sets of specific, still undiscovered natural substrates. Genes encoding class II HDACs 7, 9 and 10 isoforms are absent in schistosomes. Similarly, HDAC11 (the single class IV member), while present in the ecdysozoan branch of invertebrates, is 
not encoded in the $S$. mansoni genome and has not been described so far in other lophotrochozoans [48].

In addition to 'classical' HDACs, five $\mathrm{NAD}^{+}$-dependent sirtuins have been identified in the $S$. mansoni genome, and phylogenetic analysis showed that they are orthologs of mammalian Sirt1, Sirt2, Sirt5, Sirt6 and Sirt7 [55,56] (see review by Lancelot et al. in this issue).

\section{Biological rationale for targeting schistosome HDACs}

As mentioned above, imbalances in epigenetic mechanisms are increasingly reported to be involved in various pathologies, including cancer genesis and progression $[23,46]$. This explains the intense attempts to target the human epigenome in order to develop anticancer therapies [57-60]. Interestingly, many human
(A) Schistosome HDACs

smHDAC1 (Smp_005210), 517 aa Class I HDAC

smHDAC3 (Smp_093280), 418 aa Class I HDAC

smHDAC8 (Smp_091990), 440 aa Class I HDAC

smHDAC4 (Smp_191310), 817 aa

Class Ila HDAC

smHDAC5 (Smp_069380), 1354 aa Class Ila HDAC

smHDAC6 (Smp_138770), 1132 aa Class Ilb HDAC Class IIb HDAC ZnF

(B)
Relationships between schistosome and human HDACs

导晊

\section{Schistosoma HDAC1 293 LLLVGGGGYTI} 295 MLMLGGGGYT I 379 LLMLGGGGYT I 293 LLWVGGGYTV I 290 LLVLGGGGYTV 333 TL I LGGGGYNF 298 TL I LGGGGYNL 296 I LMNTSGGYQK IV 1173 IVLALEGGYSP 589 VVLSLEGGYDM 856 I VLALEGGHDL 999 VVLALEGGHDL Ila 906 VVLALEGGHDL 837 VVLALEGGHDL 374 LVLALEGGYQ। 374 LVLALEGGYQI 354 I I L I LEGGYNL IIb 378 L I LSLEGGYNL 297 VCAVLEGGYHL

(C)

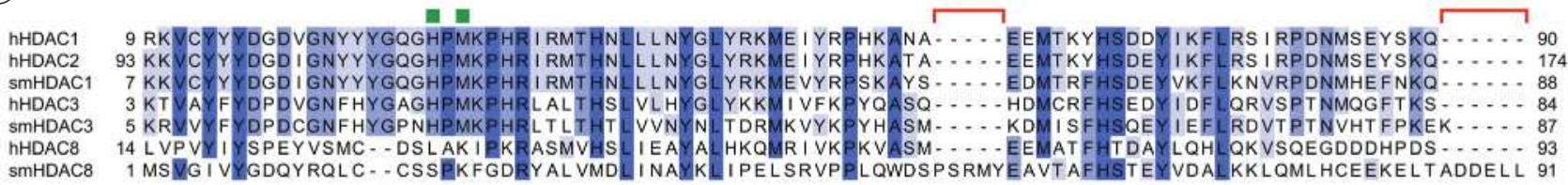
SMHDAC8 1 MS WG I VYGDQYRQLC - - CSSEKFGDRYAL VMDL I NAYKL IPELSRVPPLQWDSPSRMYEAV

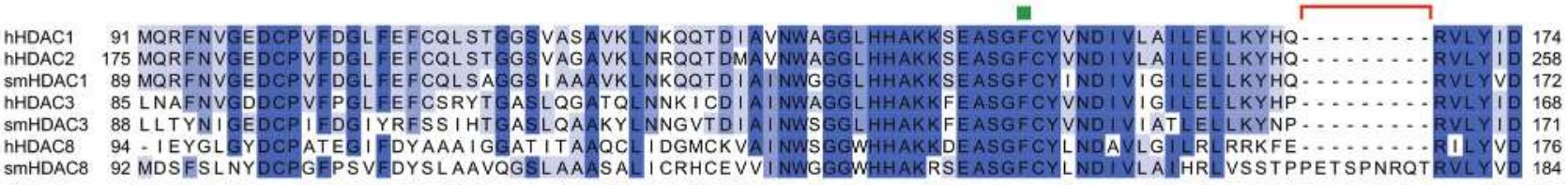
SMHDAC8 92 MDSFSLNYDCPGEPSVEDYSLAAVQGSLAAASAL I CRHCEVVI NWGGOWHHAKRSEASGFCYLND IVLAI HRLVSSTPPETSPNRQTRVLYVD 184

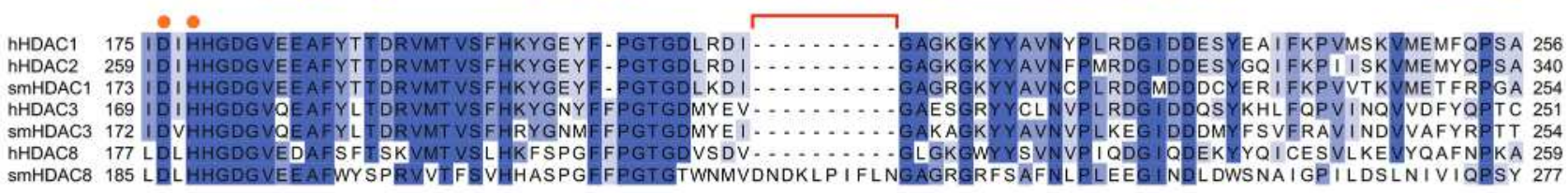

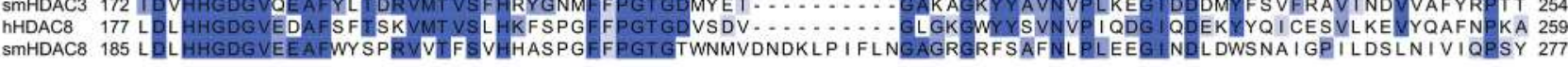

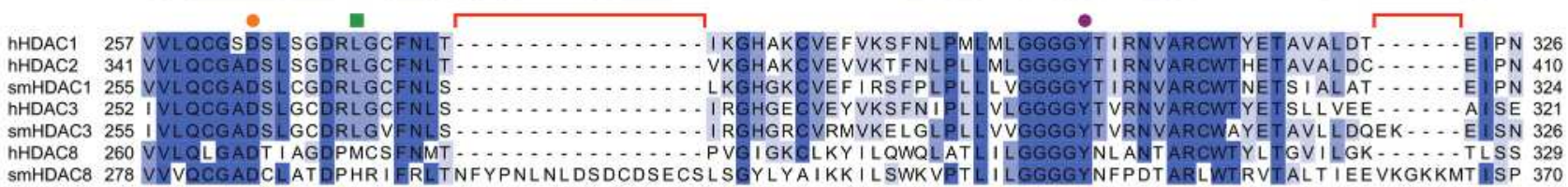

hHDAC1 327 ELPYNDYFEYFGPDFKLHISPSNM-TNONTNEYLEKIKQRLFENLRMLPHAPGVQMQAIPEDAIPEESGD- - EDEDDPDKRISICSSDKRIA 415 hHDAC2 411 ELPYNDYFEYFGPDFK L ISPSNM-T NQNTPEYMEK IKQRLFENLRMLPHAPGVQMQA IPEDAVHEDSGD... EDGEDPDKR IS IRASDKR I A 499 SMHDAC1 325 DLPYND Y YEYFGPDFK H ISPSNM-ANQNTNEYLEH I KT KLFENLRMIPHCPS VQMQD IPDDI VDFDENDAVAKDLADPDKR IS IMAADKA IM 416 hHDAC3 322 ELPYSEYFEYFAPDFT HPDVSTR I ENQNSRQYLDQIRQT I FENL KML NHAPS VQI HDVPADLLTYDRTDEADAEERGPEENYSRPEAPNEFY 414 SMHDAC3 327 ELPYSPY IEFFYPDYT HPDLTTKLDNANTRQY IEALRMTVHDNLKQL VHAPS VQFTDVPNDYLSNFCNDSNDEREKPNEMFVEEEQDSTAA- 418

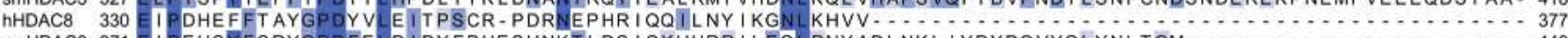

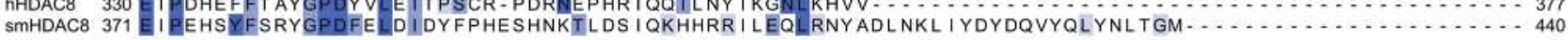


Figure 4. Repertoire of schistosome zinc-dependent histone deacetylases, and their sequence relationships to their human counterparts (see facing page). (A) Schematic representation of all schistosome HDACs including three class I HDACs (smHDAC1, 3 and 8), and three class II HDACs (smHDAC4, 5 and 6). Names in parentheses are gene ID according to the $S$. mansoni genome annotation. Note that the smHDAC6 contains two class II HDAC domains and a zinc finger (ZnF) domain located in the carboxy-terminal end. (B) Neighborjoining phylogenetic tree displaying relationship between schistosome and human HDACs. The partial alignment of HDAC sequences accompanying the phylogenetic grouping depicts a position of a catalytically essential tyrosine residue (green arrowhead) in class I and class IIb human HDACs. While this tyrosine residue is replaced by a histidine in human class Ila HDACs, schistosome class Ila HDACs (smHDAC4 and smHDAC5) retain the tyrosine residue at this position. (C) Sequence alignment of schistosome and human class I HDACs catalytic domains. The residues coordinating the zinc ion (orange circles) and the catalytically essential tyrosine residue (violet circle) are depicted. In addition, key residues that participate in the specificity of the Schistosoma HDAC8 (smHDAC8) active-site pocket are labelled with green small squares. Six insertions occurring in the smHDAC8 are depicted with red lines over the aligned sequences. For clarity, some $\mathrm{N}$-terminal and C-terminal residues were removed from the alignment.

HDAC: Histone deacetylase.

parasites, including schistosomes, share some characteristics with tumors, including high metabolic activity, a dependence on lactate fermentation as an energy source within the human host, uncontrolled (by the host) cell division and a degree of invisibility to the host immune response. It is therefore expected that an approach targeting the schistosome epigenome would be successful in treating schistosomiasis [27-28,48,50,61].

So far, only S. mansoni class I HDACs (smHDAC1, smHDAC3 and smHDAC8) have been cloned and characterized [47]. Transcriptomic experiments showed that these class I HDACs are expressed at all schistosome life-cycle stages, with smHDAC8 transcripts always being most abundant [47]. This finding was quite surprising, because normal levels of $H D A C 8$ transcripts are generally lower than those of $H D A C 1$ and $H D A C 3$ in human cells, with the exception of some cancers, where $H D A C 8$ expression is often strikingly upregulated [62]. Functional analyses have proved that smHDAC1 can repress transcriptional activity in mammalian cells, and that this activity is dependent on its catalytic activity since transcription is partially restored by treatment with a small-molecule HDAC inhibitor (HDACi) such as trichostatin A (TSA), and that a catalytic site mutant fails to repress gene transcription [47].

Although named 'histone deacetylase 8', thus far no histone protein has been identified as a protein substrate of HDAC8. For this reason, the biological role of HDAC8 has long remained elusive. A fundamental breakthrough was provided by Deardorff and coworkers [63], who demonstrated that human HDAC8 (hHDAC8) is involved in deacetylation of the cohesin complex, precisely its SMC3 subunit, and that mutations in $h H D A C 8$ gene are linked with the Cornelia de Lange syndrome. A more recent study discovered additional novel cellular protein substrates of HDAC8, including the tumor suppressor ARID1A [64].

The abundance of smHDAC8 transcript in all schistosome life-cycle stages [47], together with the fact that this enzyme contains six relatively large insertions in the protein sequence (Figure 4), indicated that the smHDAC8 could be a suitable drug target. These assumptions were further supported by biochemical and in vivo assays [27]. Enzymatic activity analyses with recombinant enzymes demonstrated that the smHDAC8 is a functional acetyl-I-lysine deacetylase exhibiting highly similar, if not identical, deacetylase activity to that observed for its human counterpart, hHDAC8 [27]. In addition, a series of single-point mutations in the smHDAC 8 active-site pocket also revealed that the catalytic mechanism of this enzyme is analogous to that seen in other eukaryotic class I HDACs [35,42-43]. More importantly, RNA interference (RNAi)-mediated downregulation of $s m H D A C 8$ expression in schistosome larvae, schistosomula, followed by their intravenous injection into mice and harvesting of surviving worms 35 days later, showed a significantly reduced worm recovery compared with mice treated with control RNA [27]. Finally, crucial roles of zinc-dependent HDACs in schistosome biology were confirmed with the use of small-molecule HDACi $[27,49,65]$.

\section{How do HDACi function as antischistosomal drugs?}

The exact mechanisms of HDACi-triggered mortality of schistosomes are not completely elucidated $[27,49,65]$. Nevertheless, recent experimental data provided evidence that HDACi have a capacity to induce a programed cell death pathway in schistosomes [49]. The effector caspases 3/7 seem to play essential roles in this HDACi-induced apoptosis. At the molecular level, it has been shown that the treatment of schistosomes with general HDACi, for example, TSA and valproic acid (Figure 2), caused a prominent accumulation of acetylated cellular proteins, more particularly of histone $\mathrm{H} 4$ whilst histone $\mathrm{H} 3$ acetylation was affected to a lesser extent [49]. More precisely, the hyperacetylated histone $\mathrm{H} 4$ protein was abundantly present in a proximal promoter region of caspase 7-coding gene upon the TSA treatment, and the presence of this hyperacetylated histone 4 was shown to be linked with increased expression of the caspase 7 gene in $S$. mansoni [49].

In addition, there is also experimental evidence that HDACi treatment affects metamorphosis of 
Schistosoma parasite. For instance, Azzi and coworkers [25] showed that the TSA inhibitor reversibly blocks transformation of $S$. mansoni larvae from the free-swimming miracidia into the intramolluskal sporocyst. Taken together, these experiments shed light on putative molecular mechanisms of $\mathrm{HDACi}$ actions that include chromatin remodeling, regulation of gene expression and programmed cell death, and which could explain the observed developmental arrest and/or mortality of HDACi-treated schistosomes.

\section{A 'piggyback' strategy to speed up the search for novel antischistosomal agents}

Development of schistosome-specific HDACi is of great pharmaceutical importance, but still remains a challenge. Various strategies can be used to search for specific inhibitors of schistosome HDACs, ranging from broad screening with large chemical libraries to structure-based inhibitor design. If the former strategy enables characterization of a large set of inhibitors, potentially with different backbones, the latter is essential in enabling the development of selective inhibitors specifically targeting the schistosomes epigenetic mechanisms.

Specifically, to speed up the search for novel antischistosomal drugs, a 'piggyback' strategy that builds on chemical scaffolds previously validated for other diseases or other targets appears of interest [66]. This 'piggybacking' of drug leads was already shown to be a valuable approach in identification and validation of new drug targets in schistosomes. For example, the use of BI-2536, which is a clinically validated selective inhibitor of human Polo-like kinase 1 (Plk1) [67], triggered profound defects in the gonads of both schistosome genders, with a strong decrease in gamete production [68]. Because schistosome Plk-1 is primarily expressed in female vitelline cells and oocytes and in male spermatocytes, the observed phenotypic defects upon the BI-2536 treatment indicated that Plk-1 represents a new drug target against schistosomiasis [68]. Some others 'piggybacking' examples in the search for new antischistosomal agents are summarized in a review by Dissous and Grevelding [66].

In human epigenetic therapy, HDACs are among the most studied epigenetic targets, and a variety of HDACi affecting cancer cells have been discovered $[57,60]$. Three of these inhibitors, suberoylanilide hydroxamic acid (SAHA or Vorinostat or Zolinza),

\section{Key terms}

'Piggybacking' of drug-leads: Chemical tailoring of drug-like small molecules (histone deacetylase inhibitors), which were successfully validated, for instance in anticancer treatments, into new antiparasitic therapeutics. cyclic depsipeptide FK228 (Romidepsin or Istodax) and Belinostat (Baleodaq) (Figure 2) have been approved by the US FDA for the treatment of refractory cutaneus T-cell lymphoma and peripheral T-cell lymphoma, and many other inhibitors are in clinical trials $[35,69]$. Nevertheless, the risk of cross-reactivity of the potential antiparasitic drugs with human (host) enzymes, which can cause off-target effects, represents a challenging issue in antiparasitic epigenome targeting strategy. To avoid this potential risk, a detailed structural knowledge of both the parasite and the host enzymes is required in order to develop and optimize chemical scaffolds that could be then safely used as antiparasitic agents.

Importantly, such a pivotal approach has recently been employed to specifically target schistosome HDAC8, where coupling of the 'piggyback' approach with structure-guided drug design yielded several drug-like molecules with promising antischistosomal activities. This achievement was enabled by the recent determination of the smHDAC8 structure at the atomic level $[27,70]$.

\section{Structure \& catalytic mechanism of smHDAC8}

Crystal structures of smHDAC8 in inhibitor-free and inhibitor-bound states have recently been determined $[27,65]$. Despite the presence of six relatively large insertions (Figure 3), smHDAC8 adopts a canonical $\alpha / \beta$ fold similar to hHDAC8 (r.m.s.d. on Ca's of 1.2 $\AA$ between the human and schistosome enzymes) [27]. More precisely, the smHDAC8 forms a single $\alpha / \beta$ domain composed of a central, eight-stranded parallel $\beta$-sheet sandwiched by $15 \alpha$-helices ( $\alpha 1-\alpha 15$ ) (Figure 5). Structural comparison showed that all the above noted insertions form extended surface loops, which are located away from the active-site pocket. This suggests that these loops do not influence the catalytic mechanism directly, but may form schistosome-specific protein/protein interaction surfaces [27].

Similarly to hHDAC8, smHDAC8 contains three ions: the central catalytic divalent zinc bound at the bottom of the active-site pocket and two monovalent potassium ions. In smHDAC8 these three ions and their coordinating residues are conserved (Figure 5). Unlike hHDAC8, smHDAC8 could be crystallized in a non-inhibited form. However, careful inspection of electronic density revealed the unambiguous presence of an L-tartrate (2,3 dihydroxysuccinic acid) molecule provided by the crystallization buffer. This L-tartrate molecule was bound in the smHDAC8 active site where it coordinated the catalytic zinc ion (Figure 4). In fact, crystals of 'apo' smHDAC8 were only obtained in presence of L-tartrate and of the related succinic 


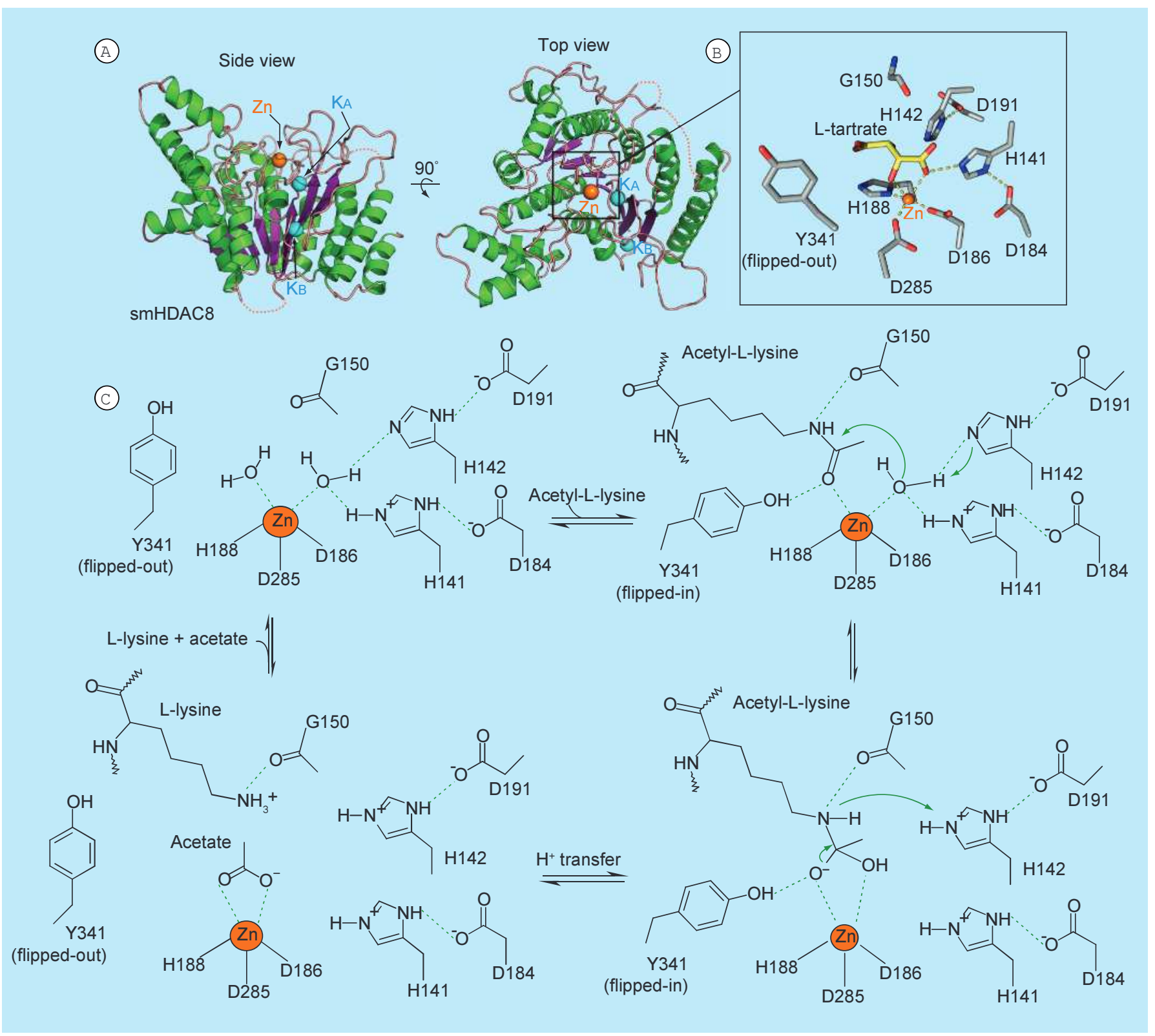

Figure 5. Structure and proposed catalytic mechanism of smHDAC8. (A) Cartoon representations of the smHDAC8 structure. Orange sphere, catalytic zinc ion; blue spheres, potassium ions ( $K_{A}$ and $K_{B}$ sites). (B) Close-up view of the smHDAC8 active site. Residues involved in catalytic mechanism are drawn as stick. The carbon atoms of proteins side chains are in gray, whereas the carbon atoms of L-tartrate are in yellow; orange sphere, catalytic zinc ion. (C) Proposed catalytic mechanism of smHDAC8.

acid molecule, only the former giving rise to welldiffracting crystals. This hints at a relative flexibility of smHDAC8 (and also hHDAC8) that can only be counteracted by an inhibitor or a small molecule binding in the active site of these enzymes via coordination with the zinc ion and hydrogen bonding with surrounding residues.

The smHDAC8 structure suggested that the catalytic mechanism of this enzyme is analogous to that proposed for other class I HDACs (Figure 5) [27,35,42]. Briefly, catalysis by the smHDAC8 is initiated by an activation of zinc-coordinated water molecule with a help of the histidine dyad (H141 and H142), and followed by a polarization of a substrate carbonyl via zinc coordination and hydrogen bonding with a catalytically essential tyrosine residue (Y341). This mediates the general base-triggered nucleophilic attack of the activated, zinc-coordinated water molecule (Figure 5). In the triggering of the nucleophilic attack, active-site histidine (H141 and H142) and aspartate (D184 and D191) side chains are expected to play crucial roles, where one of the histidines functions as general base 


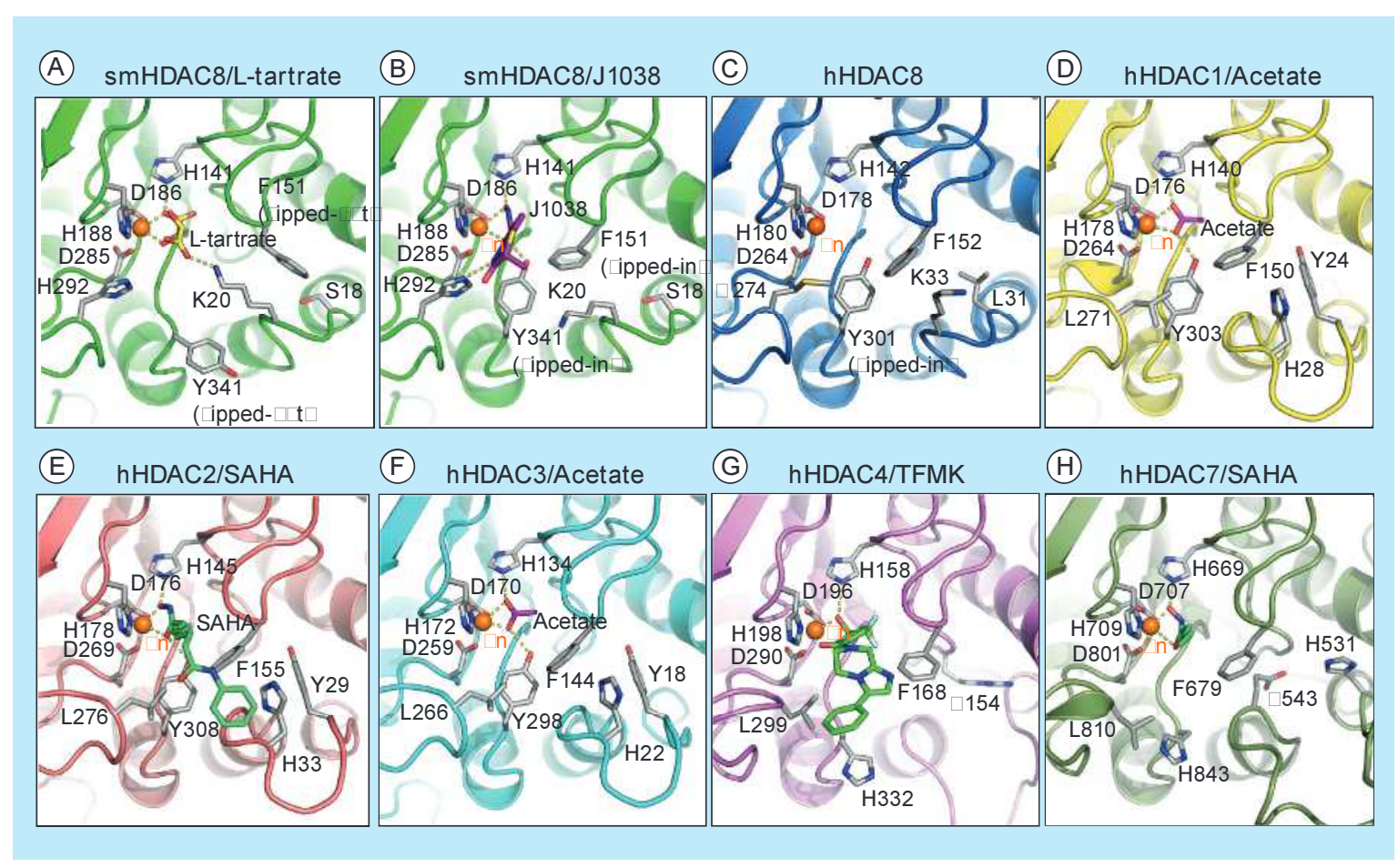

Figure 6. Unexpected structural plasticity observed in the active-site cleft of the smHDAC8 enzyme. Ribbon representation of the active site of (A) smHDAC8 occupied by the small-molecule L-tartrate, (B) smHDAC8 inhibited with the J1038 inhibitor, (C) hHDAC8 inhibited with largazole (for clarity the largazole molecule was removed from the picture), (D) hHDAC1 with a bound acetate molecule, (E) hHDAC2 blocked with the SAHA inhibitor, (F) hHDAC3 with a bound acetate molecule, (G) hHDAC4 blocked with a trifluoromethylketone (TFMK)based inhibitor and (H) hHDAC7 blocked with the SAHA inhibitor. Residues participating in zinc binding, catalysis and active-site formation are shown as sticks. Importantly, note that smHDAC8 F151 can adopt both the flippedout and flipped-in conformations (panels $A$ and $B$ ), whilst corresponding residues in all human HDAC structures determined so far adopt only the flipped-in conformation, because of various steric hindrances (panels $\mathrm{C}$ to $\mathrm{H}$ ).

(Figure 5). The resulting tetrahedral intermediate is stabilized by coordination to the zinc and hydrogen bonding with Y341 and G150, and residue H142 likely functions as a proton donor that facilitates collapse of the tetrahedral intermediate. The acetate molecule, product resulting from the reaction, coordinates the zinc ion, and is subsequently removed from the active site. While it is widely believed that the removal of acetate is transported through an internal exit channel in hHDAC8, this exit path seems to be inexistent in smHDAC8, suggesting an alternative way to remove the acetate product [27].

\section{Zooming into the smHDAC8 active site reveals key druggable specificities}

Comparison of the active sites of smHDAC8 and its human counterpart reveals several major changes between these proteins. An expected feature is the replacement of hHDAC8 M274 by $\mathrm{H} 292$ in smHDAC8 that diminishes the hydrophobic character of the pocket that normally accommodates the aliphatic part of the incoming acetylated lysine (Figure 6). The second structural difference concerns the flipping of the smHDAC8 F151 side chain that can be either turned away from the catalytic pocket (flipped-out conformation) or turned toward the active-site pocket (flipped-in conformation) (Figure 6). The adoption of the flipped-out conformation of the smHDAC8 F151 seems to be exclusively a schistosome-specific feature, since no similar flipping of this highly conserved phenylalanine residue was observed in any other HDAC and HDAC-related metalloenzyme structures determined so far (Figure 6) [42-43,71-75].

The explanation for this unusual conformation of the smHDAC8 F151 side chain resides in the absence of residues causing steric hindrance in loops surrounding the active-site pocket. For instance, in hHDAC8 the F152 residue cannot adopt the flipped-out conformation, because it is incompatible with the conformation of the L31 side chain that is itself locked in this conformation by its surrounding neighbors (Figure 6). In the schistosome HDAC8 enzyme, this leucine is replaced by a serine (S18). This smaller residue enlarges the pocket accommodating the F151 side chain, enabling it to adopt its observed flipped-out conformation (Figure 6). This conformation of F151 
appears favored over its flipped-in conformation since it contributes to strong Van der Waals contacts in the pocket where it is bound.

Careful investigation of human HDAC1, HDAC2 and HDAC3 structures, which are also class I HDACs, showed that the flipped-in conformation is also constrained in these HDACs due to the position of an invariant tyrosine provided by a specific loop of HDAC1, HDAC2 and HDAC3 (Y24, Y29 and Y18, respectively) (Figure 6). In members of human class II HDACs, HDAC4 and HDAC7, a flipped-out conformation of this phenylalanine would bring its side chain into a strongly unfavorable close vicinity of charged residues (hHDAC4 R154 and hHDAC7 H531 and E543), which also affect the hydrophobic character of the pocket used to accommodate the flipped-out conformation of the phenylalanine residue (Figure 6). The functional reason for this favored flipped-out conformation is unclear and could hint to a different, possibly bulkier substrate for smHDAC8 than hHDAC8. Alternatively, this flipping-out of F151 could enable the removal of the acetate product of the deacetylation reaction.

Another structural feature that distinguishes $\mathrm{smH}$ DAC8 from its human counterpart is the position of the lysine 20 (K20) side chain. As shown in Figure 6, the crystal structure of non-inhibited smHDAC8, where an L-tartrate molecule coordinates the catalytic zinc ion, reveals that $\mathrm{K} 20$ interacts with the L-tartrate molecule in the active-site pocket. The access of the K20 residue to the catalytic pocket is allowed by the flippedout conformation of the F151 residue. When the F151 adopts the flipped-in conformation, as is observed in the case of the smHDAC $8 / \mathrm{J} 1038$ complex structure, the K20 side chain is unable to enter the active-site pocket (Figure 6). Accordingly, this lysine is conserved in hHDAC8 (Figure 4), but is prevented from entering the active site due to the fixed flipped-in conformation of F152 (Figure 6). This feature represents an additional structural specificity of the smHDAC 8 catalytic pocket, and potentially offers another specific asset for the development of schistosome-specific HDAC8 inhibitors.

In conclusion, the flipped-out conformation of smHDAC8 F151 appears highly specific to the schistosome enzyme in contrast to human HDACs, suggesting that this feature, together with the specific replacement of human M274 by schistosome H292 and potentially the positioning of the K20 side chain, provides the basis for the design of specific inhibitors targeting smHDAC8. Specifically, the flippedout conformation of F151 creates a broader catalytic pocket in smHDAC8 that should be able to accommodate bulkier inhibitors as compared with human HDACs [27].

\section{On the track for selective inhibitors of schistosome HDAC8}

To date, a large number of structurally diverse $\mathrm{HDACi}$ have been developed [42,57,59]. A common feature of the first discovered HDACi was a lack of any particular selectivity toward a specific isoform. Some of compounds are therefore known to be 'pan'-HDACi, while more recent inhibitors exhibit class- or isoform-selectivity [35]. The HDACi typically share a well-recognized pharmacophore that consists of three parts: a zinc-binding warhead that is buried deep in the catalytic pocket of the HDAC enzyme to chelate the catalytic zinc and interacts via hydrogen bonding with the surrounding active-site residues; a linker that passes through the hydrophobic channel of HDACs; and an exposed capping group that interacts with the rim region, where individual HDAC isoforms exhibit remarkable structural variation and conformational plasticity [76].

Up to now, several hHDAC8-selective inhibitors have been discovered. The first compound exhibiting $>200$-fold selectivity over other human HDAC isoforms was an indole-based hydroxamic acid inhibitor, PCI-34051 (Figure 2) [77]. This compound has been shown to specifically induce apoptosis at low micromolar concentrations in cells derived from T-cell lymphomas such as Jurkat and HuT78 cells [77]. Recent attempts yielded to the development of many other compounds that show selectivity toward the HDAC8 isoform [76,78]. However, no crystallographic data are currently available that could provide the molecular basis by which these compounds specifically inhibit the HDAC8 isoform.

Based on the crystal structure of smHDAC8 in 'apo' form, a virtual screening search for new inhibitor scaffolds that would fit into the enlarged catalytic pocket of smHDAC8 has been performed [27,70]. The large initial set of scaffolds identified by chemical virtual screening was further analyzed by biochemical and biophysical assays. As an outcome, two highestranked linker-less hydroxamate-based scaffolds, J1038 (2-methyl-3-oxo-4H-1,4-benzothiazine-6-carbohydroxamic acid) and J1075 (3-chlorobenzothiophene2-carbohydroxamic acid) (Figure 2), were identified. Instead of an aliphatic linker connecting the zincbinding hydroxamate head and the hydrophobic capping group, an otherwise typical feature for many 'pan'-HDACs inhibitors such as SAHA, M344 and

\section{Key terms}

Virtual screening: Computational approach used in drug discovery process to search libraries of small molecules in order to identify chemical scaffolds that should strongly bind to a target, for example a parasitic enzyme. 


\begin{tabular}{|c|c|c|c|c|c|}
\hline Inhibitor & $\begin{array}{l}\text { smHDAC8 } \\
\text { inhibition }\end{array}$ & $\begin{array}{l}\text { hHDAC8 } \\
\text { inhibition }\end{array}$ & $\begin{array}{l}\text { hHDAC1 } \\
\text { inhibition }\end{array}$ & $\begin{array}{l}\text { hHDAC3 } \\
\text { inhibition }\end{array}$ & $\begin{array}{l}\text { hHDAC6 } \\
\text { inhibition }\end{array}$ \\
\hline$J 1075$ & $4.3 \pm 0.3 \mu \mathrm{M}$ & $3.1 \pm 0.5 \mu \mathrm{M}$ & $11.6 \pm 3.5 \mu \mathrm{M}$ & $12.5 \pm 1.5 \mu \mathrm{M}$ & $1.3 \pm 1.2 \mu \mathrm{M}$ \\
\hline$J 1038$ & $1.5 \pm 0.4 \mu \mathrm{M}$ & $1.0 \pm 0.1 \mu \mathrm{M}$ & $14.9 \pm 1.3 \mu \mathrm{M}$ & $12.3 \pm 0.7 \mu \mathrm{M}$ & $3.5 \pm 0.5 \mu \mathrm{M}$ \\
\hline SAHA & $1.6 \pm 0.2 \mu \mathrm{M}$ & $0.4 \pm 0.1 \mu \mathrm{M}$ & $117 \pm 5.6 \mathrm{nM}$ & $92 \pm 12 \mathrm{nM}$ & $104 \pm 8.6 \mathrm{nM}$ \\
\hline M344 & $3.1 \pm 0.5 \mu \mathrm{M}$ & $0.3 \pm 0.03 \mu \mathrm{M}$ & $43 \pm 4.9 \mathrm{nM}$ & $64 \pm 6 n M$ & $17.6 \pm 4 \mathrm{nM}$ \\
\hline DS45 & $50 \pm 4 \mu \mathrm{M}$ & $200 \pm 37 \mu \mathrm{M}$ & ND & ND & ND \\
\hline
\end{tabular}

TSA (Figure 2), these two newly discovered inhibitors only contained annellated ring systems [27].

Importantly, the J1075 and J1038 compounds showed a significant drop in inhibition rates for major human HDACs (Table 1), while inducing apoptosis and mortality of schistosomes [27]. The crystal structures of these inhibitors bound to smHDAC8 revealed that these inhibitors coordinate the catalytic zinc with their hydroxamate moieties in a fashion highly similar to 'pan'-HDACs inhibitors; however, their binding to smHDAC8 used otherwise different binding modes. Specifically, the annellated ring systems of both inhibitors adopt perpendicular conformations when bound to smHDAC8 (Figure 7).

More precisely, J1075 binding forces the F151 residue to remain in its flipped-out conformation, but does not interact with the schistosome-specific $\mathrm{H} 292$ residue at the rim of the catalytic pocket. In addition, due to the presence of a bulkier chlorine atom in J1075, this inhibitor prevents Y341 from adopting its catalytic flipped-in conformation. In fact, in this structure Y341 adopts an intermediate conformation between the ones observed in the native (non-inhibited) and inhibitor-bound forms of smHDAC8 (Figures 6 \& 7). In the smHDAC8/ J1038 complex, the J1038 inhibitor forms a hydrogen bond with the side chain of smHDAC8-specific H292, thus using another structural specificity of this enzyme (Figure 7). However, binding of J1038 induces the F151 to adopt its flipped-in conformation [27].

Of note, these newly identified smHDAC8 linkerless inhibitors (J1075 and J1038) share some characteristics with hydroxamate-based inhibitors of matrix metalloproteinase [79], which might cause off-target problems. As for generic HDACi, it will be important to modify these initial scaffolds into more potent and specific inhibitors by adding chemical capping groups

\section{Key term}

Focused chemical library: Series of chemicals (inhibitors) known to block catalytic activity of a particular enzyme family such as histone deacetylases. that will interact with sub-pockets at the rim of the smHDAC8 active site.

In parallel to this work, focused chemical library screening with recombinant smHDAC8 enzyme identified a mercaptoacetamide inhibitor, referred as DS45 (Figure 2) in this review. This compound inhibited smHDAC8 activity in the micromolar range, showed fourfold selectivity over its human counterpart, hHDAC8 (Table 1), and triggered apoptosis in in vitro cultured schistosomes in a dose-dependent manner [65]. Mercaptoacetamide-based compounds have been previously shown to be potent inhibitors of class II HDACs, with promising positive effects on learning and memory in a mouse model of Alzheimer's disease [80]. The crystal structure of schistosome HDAC8 complexed with the thiol DS45 shed light on the inhibition mode of mercaptoacetamide-based inhibitors (Figure 7). Specifically, the DS45 inhibitor was found to be perfectly accommodated in the catalytic pocket, where it interacts with both the catalytic zinc and the flipped-in Y341 residue (Figure 7) via its mercaptoacetamide group. The zinc ion coordination geometry in the smHDAC8/DS45 structure is tetrahedral and similar to that observed in the hHDAC8/largazole structure [81]. Interestingly, upon DS45 binding, the smHDAC8 F151 residue adopts a flipped-in conformation, and together with F216, forms the hydrophobic tunnel that accommodates the slightly kinked aliphatic linker of the inhibitor (Figure 7). The smHDAC8/ DS45 complex appears as the first crystal structure of any HDAC inhibited by a mercaptoacetamide inhibitor, providing the molecular basis for further design of inhibitors bearing such a warhead.

\section{Intrinsic HDAC8 flexibility as a hurdle in the development of high-affinity inhibitors}

hHDAC8 has only been crystallized in the presence of inhibitors or a peptidic substrate, suggesting that native hHDAC8 contains flexible parts, which may prevent crystallization. Similarly, smHDAC8 was crystallized in a non-inhibited form, but during the crystallization 


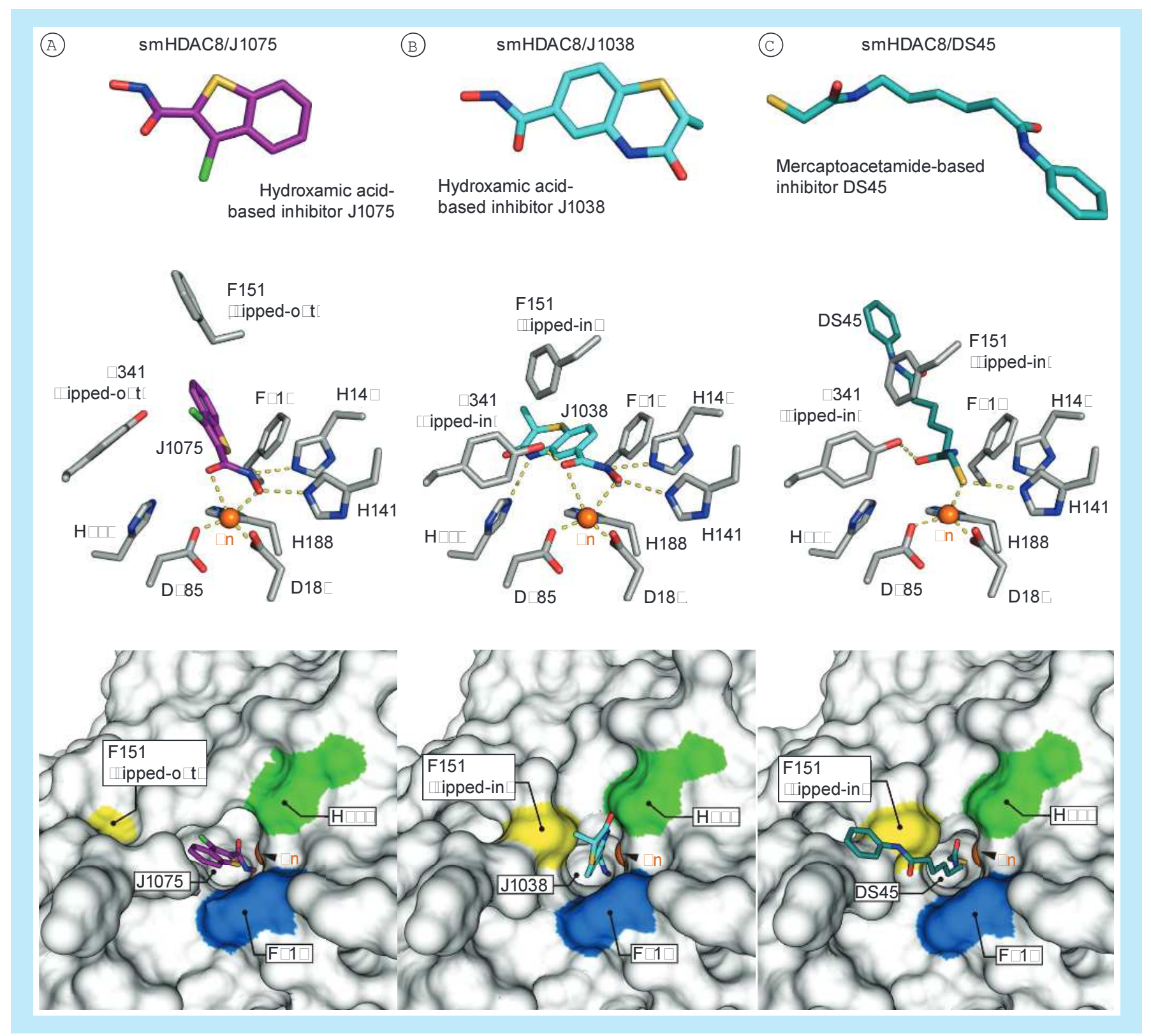

Figure 7. Structural mechanisms of smHDAC8 inhibition via small-molecule inhibitors identified by structure-based approach and focused library screening. Inhibitor structures (top panels), close-up views of the active site (middle panels) and surface representations (bottom panels) of smHDAC8 inhibitions by (A) linker-less hydroxamic acid-based inhibitor J1075, (B) linker-less hydroxamic acid-based inhibitor J1038 and (C) mercaptoacetamide inhibitor DS45. Note that the binding of J1075 to smHDAC8 keeps the F151 side chain in its flipped-out conformation, whilst the binding of J1038 and DS45 triggers F151 to adopt a flipped-in conformation. In addition, J1038 interacts via a hydrogen bond with schistosome-specific residue, H292.

process an L-tartrate molecule, present in the crystallization buffer, occupied the enzyme's active site resembling inhibitor binding (Figure 5). These observations argue for an intrinsic flexibility of the HDAC8 active site which might be important for substrate recognition and/or catalytic activity. Thermodynamic studies further supported this possibility [82].

The different crystal structures of HDAC8 enzymes provide some clue to its flexibility. For instance, in the case of $\operatorname{smHDAC} 8$ the presence of S18 in this HDAC replacing L31 in hHDAC8 allows F151 to adopt two different conformations. In hHDAC8 structures, some loop movements at the rim of the active site are also observed [83,84]. But it is expected that flexibility of HDAC8 enzymes is even stronger. Inhibitors J1038 and J1075, which were thought through modeling studies to be poor inhibitors of hHDAC8, showed only a mild increase of $\mathrm{IC}_{50}$ values, compared with pan-HDACi, 
for hHDAC8. This increase was much stronger for the other class I HDACs, HDAC1 and HDAC3 [27].

Another aspect, which has not been taken into account in these studies so far, is the possible implication of regulatory subunits on the activity of HDACs. For instance, human HDAC1 and HDAC 3 are known to be part of large complexes and their activity is increased and modulated by their binding to protein partners [71,72]. Surprisingly, these studies also revealed the importance of inositol- $(1,4,5,6)$-tetrakisphosphate (IP4) in stabilizing these interactions and modulating the HDAC activity. Specifically, IP4 was shown to interact with loops at the rim of HDAC3 active site [72], possibly selecting for a catalytically productive conformation of the enzyme. In the case of hHDAC8, only few partners of this enzyme have been identified. It is therefore quite likely that this enzyme does not belong to a large complex and that its activity is not modulated by other subunits. In the case of the schistosome HDAC8 enzyme, the existence of protein partners has never been investigated. Yet, the presence of large schistosome-specific loops in the smHDAC8 enzyme [27] suggests that it can interact specifically with other proteins that will possibly regulate its activity. This possibility calls therefore for a comparative evaluation of the effects of the inhibitors in vivo both in human cells and in schistosomes.

As such, the inherent flexibility of HDAC8 enzymes represents a hurdle for the rational development of high-affinity parasite-specific inhibitors. Specifically, in the case of smHDAC8, a vast majority of HDACi tested against this enzyme showed significantly higher $\mathrm{IC}_{50}$ values compared with hHDAC8. For instance, the highly potent and isoform-selective compound PCI-34051 [77] is a more than 10-fold weaker inhibitor of smHDAC8 activity compared with its human counterpart hHDAC8 [65]. Yet, the characterization of an inhibitor, DS45, that shows a lower $\mathrm{IC}_{50}$ value for smHDAC8 than hHDAC8 [65] highlights the possibility of obtaining smHDAC8-specific inhibitors by designing molecules that not only make use of the specific physico-chemical properties of the active site of this enzyme, but also build on specific interactions made by these inhibitors with residues/pockets at the rim of the active site.

Interestingly, these different aspects replace the inhibition studies of other HDACs from schistosomes in a different perspective. Notably, unpredicted structural differences and intrinsic flexibility could be used for the design of schistosome-specific drug-leads despite a high sequence homology between the human and schistosome enzymes. Here again, a structure-based approach using a 'piggyback' strategy would be beneficial for the search toward improved antiparasitic agents.

\section{Next-generation HDACi: a 'pan- antihelminthic' therapy on the horizon?}

Biochemical and structural characterization of $S$. mansoni HDAC8 revealed broader pharmaceutical implications. Notably, sequence comparison showed that smHDAC8 S18, which enables F151 flipping out, is conserved in all schistosome HDAC8 enzymes sequenced so far, including $S$. japonicum and $S$. haematobium, as well as another trematode species, Clonorchis sinensis. The same is also true for smHDAC8 H292. Structural modeling of the HDAC8 structures from these different parasitic species based on sequence alignment and smHDAC8 structure template did not suggest any amino acid substitution in these enzymes that could prevent, directly or indirectly, F151 to adopt its flipped-out conformation [27]. These observations are of high importance since they imply that inhibitors specifically targeting smHDAC8 should also inhibit the corresponding enzymes of S. japonicum, S. haematobium and $C$. sinensis, and possibly those of other trematode species that affect many populations worldwide. Moreover, S. haematobium and $C$. sinensis are also linked with the progression of cancer [85]. Hence, development of drugs fighting these infections should also have an implication in cancer prevention.

Prominently, smHDAC8 S18 but also smHDAC8 $\mathrm{H} 292$ are preserved in the HDAC8 sequences of the cestodes Echinococcus multilocularis, Echinococcus granulosus and Taenia solium [86] that also cause serious human diseases. Structural models of these HDAC8 proteins from E. multilocularis, E. granulosus and T. solium revealed that the active sites of these enzymes are conserved compared with that of smHDAC8. Specifically, as for the various schistosome species, the flipped-out conformation of the phenylalanine corresponding to smHDAC8 F151 should also be conserved in these enzymes. In addition, the position of the histidine corresponding to smHDAC8 $\mathrm{H} 292$ should be the same as in the schistosome enzyme [27]. These observations suggest that the selective inhibitors developed against smHDAC8 could be used in a pan-antihelminthic therapy.

\section{Conclusions}

Enormous contemporary interest in epigenetic therapies, notably in targeting of metal-utilizing HDACs, resulted in the development of a wide range of druglike HDACi. In this review, we summarized current progresses in the development of small-molecule inhibitors targeting zinc-dependent HDACs from schistosomes. From this work, it appears obvious that structural characterizations at high resolution combined with computational approaches such as docking and binding free energy calculation represent valuable 
tools in the discovery of drug-leads specifically targeting parasites' epigenetic machinery, as shown for the schistosome HDAC8 enzyme. Thus, structure-guided optimization of the lead inhibitors may result in a development of schistosome-specific HDACi that can be used as antiparasitic drugs. Nevertheless, there are still many challenges that have to be clarified if such agents are to be used in clinical trials.

Among these issues belong side effects and bioavailability of HDACi. Most HDACi that are currently approved for use in humans, or are in clinical trials are, at best, moderately selective for an HDAC class and not for one particular enzyme. In an antiparasitic epigenome targeting strategy, it is essential to maximize the selectivity of the inhibitors developed in order to avoid possible side effects that can be associated with treatment with HDACi. For example, side effects of treatment with SAHA (Vorinostat or Zolinza) include fatigue, nausea and diarrhea, but the most severe adverse effect observed with HDACi has been cardiac toxicity, including ventricular arrhythmia [87]. However, the dosing schedule for HDACi in cancer therapy usually involves multiple doses given over a long period. The objective for schistosomiasis therapy is to develop a single-dose strategy, as is the case for praziquantel. Together with selectivity for the schistosome enzyme over human HDACs, this should ensure that the side effects of treatment are minimal.

\section{Future perspective: from HDAC drug-leads to new antiparasitic agents}

A huge progress in semi-automation of phenotypic screening and drug discovery methodologies for schistosomiasis was achieved over the last years [88]. However, little is still known about bioavailability and stability of HDACi in the animal model of schistosomiasis. So far, most studies investigating the possibility of the use of HDACi for the treatment

\section{Key term}

Phenotypic screening: Automated or semi-automated workflows for the discovery of new antiparasitic agents such as small molecules that alter the phenotype of a parasitic organism in a desired manner.

of schistosomiasis worked entirely with schistosome parasites cultured in vitro $[27,65]$. Testing of $\mathrm{HDACi}$ in the animal model of infection should bring new insights into the control of schistosomes via HDACi, and will in fact be required before considering HDACi for preclinical trials.

Collectively, the continued discovery and development of potent and selective small-molecule $\mathrm{HDACi}$ should bring new therapeutic agents not only for helmithic infections, but also for a spectrum of other human parasitic diseases, among others malaria, leishmaniasis, Chagas disease, toxoplasmosis and trypanosomiasis. Clearly, these developments targeting HDACs also fully apply to the numerous epigenetic players found in parasites, providing a wealth of potential pharmaceutical targets for the future.

\section{Financial \& competing interests disclosure}

This work and the authors of this manuscript have been supported by funding from the European Union's Seventh Framework Program for research, technological development and demonstration under grant agreements no's. 241865 (SEtTReND) and 602080 (A-ParaDDisE). C.R., M.M. and R.J.P. are supported by institutional funds from the Centre National de la Recherche Scientifique (CNRS), the Institut National de la Santé et de la Recherche Médicale (INSERM), the Université de Strasbourg and the Université de Lille 2, the French Infrastructure for Integrated Structural Biology (FRISBI; ANR10-INSB-05-01) and by Instruct as part of the European Strategy Forum on Research Infrastructures (ESFRI). The hHDAC8 research of M.J. (JU 295/13-1) and W.S. (SI 868/13-1) is funded by the Deutsche Forschungsgemeinschaft (DFG). G.O.

\section{Executive summary}

- Eukaryotic pathogens frequently transform into many phenotypically distinct stages during their complex life cycles. (Morphological transformations of eukaryotic parasites)

- Epigenetic plasticity drives these pathogens' morphogenetic and developmental programs. (Important role of epigenetic regulations in parasites development)

- Chemical control of epigenetic pathways represents an Achilles' heel of eukaryotic parasites. (Targeting epigenetic players in eukaryotic parasites)

- Zinc-dependent histone deacetylases (HDACs) are pharmaceutically highly investigated targets for epigenetic therapy. (Drugging HDACs for epigenetic therapy)

- 'Piggybacking' of HDAC inhibitors previously developed for anticancer treatments into new antiparasitic therapeutics speeds up the drug discovery process. ('Piggybacking: repurposing drugs)

- Structural analyses at high resolution combined with virtual screening represent valuable tools in the development of novel potent and selective HDAC inhibitors with antiparasitic properties. (Structure-based drug design)

- Targeting of epigenetic enzymes is a valid approach to treat diseases caused by eukaryotic pathogens. (Future perspective of anti-parasitic epigenome strategy) 
is supported by CNPq (309312/2012-4, 403049/20121) and FAPEMIG (PPM-00439-10). The authors have no other relevant affiliations or financial involvement with any organization or entity with a financial interest in or financial conflict with

\section{References}

Papers of special note have been highlighted as:

- of interest; $\cdots$ of considerable interest.

1 Brown M. Schistosomiasis. Clin. Med. 11(5), 479-482 (2011).

2 Ross A, Bartley P, Sleigh A et al. Schistosomiasis. N. Engl. J. Med. 346(16), 1212-1220 (2002).

3 Barsoum R. Urinary schistosomiasis: review. J. Adv. Res. 4(5), 453-459 (2013)

4 Elbaz T, Esmat G. Hepatic and intestinal schistosomiasis: review. J. Adv. Res. 4(5), 445-452 (2013).

5 Gray D, Ross A, Li Y, Mcmanus D. Diagnosis and management of schistosomiasis. BMJ 342, d2651 (2011).

6 Dömling A, Khoury K. Praziquantel and Schistosomiasis. ChemMedChem 5(9), 1420-1434 (2010).

7 Doenhoff M, Cioli D, Utzinger J. Praziquantel: mechanisms of action, resistance and new derivatives for schistosomiasis Curr. Opin. Infect. Dis. 21, 659-667 (2008).

8 Doenhoff M, Kusel J, Coles G, Cioli D. Resistance of Schistosoma mansoni to praziquantel: is there a problem? Trans. R. Soc. Trop. Med. Hyg. 96(5), 465-469 (2002).

9 Coeli R, Baba E, Araujo N, Coelho P, Oliveira G. Praziquantel treatment decreases Schistosoma mansoni genetic diversity in experimental infections. PLoS Negl. Trop. Dis. 7(12), e2596 (2013).

10 Conaway J. Introduction to theme 'chromatin, epigenetics, and transcription'. Annu. Rev. Biochem. 81(1), 61-64 (2012).

11 Arrowsmith C, Bountra C, Fish P, Lee K, Schapira M. Epigenetic protein families: a new frontier for drug discovery. Nat. Rev. Drug Dis. 11(5), 384-400 (2012).

12 Luger K, Mader A, Richmond R, Sargent D, Richmond T. Crystal structure of the nucleosome core particle at $2.8 \AA$ resolution. Nature 389(6648), 251-260 (1997).

13 Li G, Reinberg D. Chromatin higher-order structures and gene regulation. Curr. Opin. Genet. Dev. 21(2), 175-186 (2011).

14 Dawson M, Kouzarides T. Cancer epigenetics: from mechanism to therapy. Cell 150(1), 12-27 (2012).

15 Chen T, Dent $S$. Chromatin modifiers and remodellers: regulators of cellular differentiation. Nat. Rev. Genet. 15(2), 93-106 (2014).

16 Margueron R, Reinberg D. Chromatin structure and the inheritance of epigenetic information. Nat. Rev. Genet. 11(4), 285-296 (2010).

17 Bannister A, Kouzarides T. Regulation of chromatin by histone modifications. Cell Res. 21(3), 381-395 (2011)

18 Kouzarides T. Chromatin modifications and their function. Cell 128(4), 693-705 (2007). the subject matter or materials discussed in the manuscript apart from those disclosed.

No writing assistance was utilized in the production of this manuscript.

19 Zentner G, Henikoff S. Regulation of nucleosome dynamics by histone modifications. Nat. Struct. Mol. Biol. 20(3), 259-266 (2013)

20 Bernstein B, Meissner A, Lander E. The mammalian epigenome. Cell 128(4), 669-681 (2007).

21 Ivanov M, Barragan I, Ingelman-Sundberg M. Epigenetic mechanisms of importance for drug treatment. Trends Pharmacol. Sci. 35(8), 384-396 (2014).

22 Murphy Tm, Mill J. Epigenetics in health and disease: heralding the EWAS era. Lancet 383(9933), 1952-1954 (2014).

23 Handel A, Ebers G, Ramagopalan S. Epigenetics: molecular mechanisms and implications for disease. Trends Mol. Med. 16(1), 7-16 (2010).

24 Gomez-Diaz E, Jorda M, Peinado M, Rivero A. Epigenetics of host-pathogen interactions: the road ahead and the road behind. PLoS Pathog. 8(11), e1003007 (2012).

25 Azzi A, Cosseau C, Grunau C. Schistosoma mansoni: Developmental arrest of miracidia treated with histone deacetylase inhibitors. Exp. Parasitol. 121(3), 288-291 (2009).

26 Coleman B, Skillman K, Jiang R et al. A Plasmodium falciparum histone deacetylase regulates antigenic variation and gametocyte conversion. Cell Host Microbe 16(2), 177-186 (2014).

27 Marek M, Kannan S, Hauser A et al. Structural basis for the inhibition of histone deacetylase 8 (HDAC8), a key epigenetic player in the blood fluke Schistosoma mansoni. PLoS Pathog. 9(9), e100364 (2013).

.. Validates schistosome HDAC8 as a relevant epigenetic drug target, and provides molecular basis for the selective inhibition of schistosome HDAC 8 .

28 Carneiro V, De Abreu Da Silva I, Torres E et al. Epigenetic changes modulate schistosome egg formation and are a novel target for reducing transmission of schistosomiasis. PLoS Pathog. 10(5), e1004116 (2014).

- Shows key roles of histone acetyltransferases (H ATs) in the production of the schistosome eggshell protein, smp14.

29 Hansen Fk, Sumanadasa Sdm, Stenzel K et al. Discovery of HDAC inhibitors with potent activity against multiple malaria parasite life cycle stages. Eur. J. Med. Chem. 82, 204-213 (2014).

30 Bougdour A, Maubon D, Baldacci P et al. Drug inhibition of HDAC3 and epigenetic control of differentiation in Apicomplexa parasites. J. Exp. Med. 206(4), 953-966 (2009).

31 Malmquist N, Moss T, Mecheri S, Scherf A, Fuchter M. Small-molecule histone methyltransferase inhibitors display rapid antimalarial activity against all blood stage forms in Plasmodium falciparum. Proc. Natl Acad. Sci. USA 109(41), 16708-16713 (2012). 
32 Olsen C. Expansion of the lysine acylation landscape. Angew. Chem. Int. Ed. Engl. 51(16), 3755-3756 (2012).

33 Choudhary C, Weinert B, Nishida Y, Verdin E, Mann M. The growing landscape of lysine acetylation links metabolism and cell signalling. Nat. Rev. Mol. Cell Biol. 15(8), 536-550 (2014).

34 Falkenberg K, Johnstone R. Histone deacetylases and their inhibitors in cancer, neurological diseases and immune disorders. Nat. Rev. Drug Dis. 13(9), 673-691 (2014).

35 Finley A, Copeland R. Small molecule control of chromatin remodeling. Chem. Biol. 21(9), 1196-1210 (2014).

36 Yang X, Seto E. HATs and HDACs: from structure, function and regulation to novel strategies for therapy and prevention. Oncogene 26(37), 5310-5318 (2007).

37 Shahbazian M, Grunstein M. Functions of site-specific histone acetylation and deacetylation. Annu. Rev. Biochem. 76(1), 75-100 (2007)

38 Josling G, Selvarajah S, Petter M, Duffy M. The role of bromodomain proteins in regulating gene expression. Genes 3(2), 320-343 (2012).

39 Filippakopoulos P, Knapp S. Targeting bromodomains: epigenetic readers of lysine acetylation. Nat. Rev. Drug Dis. 13(5), 337-356 (2014).

40 Gregoretti I, Lee Y, Goodson H. Molecular evolution of the histone deacetylase family: functional implications of phylogenetic analysis. J. Mol. Biol. 338(1), 17-31 (2004).

41 De Ruijter A, Van Gennip A, Caron H, Kemp S, Van Kuilenburg A. Histone deacetylases (HDACs) characterization of the classical HDAC family. Biochem. J. 370, 737-749 (2003).

42 Lombardi P, Cole K, Dowling D, Christianson D. Structure, mechanism, and inhibition of histone deacetylases and related metalloenzymes. Curr. Opin. Struct. Biol. 21(6), 735-743 (2011).

43 Vannini A, Volpari C, Filocamo G et al. Crystal structure of a eukaryotic zinc-dependent histone deacetylase, human HDAC8, complexed with a hydroxamic acid inhibitor. Proc. Natl Acad. Sci. USA 101(42), 15064-15069 (2004).

44 Moniot S, Weyand M, Steegborn C. Structures, substrates, and regulators of mammalian sirtuins and opportunities and challenges for drug development. Front. Pharmacol. 3, 16 (2012).

$45 \mathrm{Xu}$ W, Li Y, Liu C, Zhao S. Protein lysine acetylation guards metabolic homeostasis to fight against cancer. Oncogene 33(18), 2279-2285 (2014).

46 Portela A, Esteller M. Epigenetic modifications and human disease. Nat. Biotechnol. 28(10), 1057-1068 (2010).

47 Oger F, Dubois F, Caby S et al. The class I histone deacetylases of the platyhelminth parasite Schistosoma mansoni. Biochem. Biophys. Res. Commun. 377(4), 1079-1084 (2008).

48 Pierce R, Dubois-Abdesselem F, Lancelot J, Andrade L, Oliveira G. Targeting schistosome histone modifying enzymes for drug development. Curr. Pharm. Des. 18(24), 3567-3578 (2012).

49 Dubois F, Caby S, Oger F et al. Histone deacetylase inhibitors induce apoptosis, histone hyperacetylation and up-regulation of gene transcription in Schistosoma mansoni. Mol. Biochem. Parasitol. 168(1), 7-15 (2009).

50 Cabezas-Cruz A, Lancelot J, Caby S, Oliveira G, Pierce R. Epigenetic control of gene function in schistosomes: a source of therapeutic targets? Front Genet 5, 10.3389 (2014).

.• Summarizes current knowledge on schistosome epigenetics and its targeting.

51 Musselman C, Lalonde M, Cote J, Kutateladze T. Perceiving the epigenetic landscape through histone readers. Nat. Struct. Mol. Biol. 19(12), 1218-1227 (2012).

52 Mireille J, Lepesant J, Grunau C, Cosseau C. Towards an understanding of the epigenetics of schistosomes: a comparative epigenomic study. Mem. Inst. Oswaldo Cruz 106(7), 823-830 (2011).

53 Brunmeir R, Lagger S, Seiser C. Histone deacetylase 1 and 2 -controlled embryonic development and cell differentiation. Int. J. Dev. Biol. 53, 275-289 (2009).

54 Lahm A, Paolini C, Pallaoro $\mathrm{M}$ et al. Unraveling the hidden catalytic activity of vertebrate class IIa histone deacetylases. Proc. Natl Acad. Sci. USA 104(44), 17335-17340 (2007).

55 Lancelot J, Caby S, Dubois-Abdesselem F et al. Schistosoma mansoni Sirtuins: characterization and potential as chemotherapeutic targets. PLoS Negl. Trop. Dis. 7(9), e2428 (2013).

- Validates schistosome Sirtuins as valuable targets for smallmolecule control of schistosomiasis.

56 Schiedel M, Marek M, Lancelot J et al. Fluorescence-based screening assays for the $\mathrm{NAD}^{+}$-dependent histone deacetylase smSirt2 from Schistosoma mansoni. J. Biomol. Screen. 20(1), 112-121 (2014).

57 West A, Johnstone R. New and emerging HDAC inhibitors for cancer treatment. J. Clin. Invest. 124(1), 30-39 (2014).

58 Feng W, Zhang B, Cai D, Zou X. Therapeutic potential of histone deacetylase inhibitors in pancreatic cancer. Cancer Lett. 347(2), 183-190 (2014).

59 Khan O, La Thangue N. HDAC inhibitors in cancer biology: emerging mechanisms and clinical applications. Immunol. Cell Biol. 90(1), 85-94 (2012).

60 Campbell R, Tummino P. Cancer epigenetics drug discovery and development: the challenge of hitting the mark. J. Clin. Invest. 124(1), 64-69 (2014).

61 Oliveira G. Cancer and parasitic infections: similarities and opportunities for the development of new control tools. Rev. Soc. Bras. Med. Trop. 47, 1-2 (2014).

62 Nakagawa M, Oda Y, Eguchi T et al. Expression profile of class I histone deacetylases in human cancer tissues. Oncol. Rep. 18(4), 769-774 (2007).

63 Deardorff M, Bando M, Nakato R et al. HDAC8 mutations in Cornelia de Lange syndrome affect the cohesin acetylation cycle. Nature 489(7415), 313-317 (2012).

64 Olson D, Udeshi N, Wolfson $\mathrm{N}$ et al. An unbiased approach to identify endogenous substrates of 'histone' deacetylase 8 . ACS Chem. Biol. 9(10), 2210-2216 (2014).

65 Stolfa D, Marek M, Lancelot J et al. Molecular basis for the antiparasitic activity of a mercaptoacetamide derivative that inhibits histone deacetylase 8 (HDAC8) from the 
human pathogen Schistosoma mansoni. J. Mol. Biol. 426(20), 3442-3453 (2014).

- Provides a structural basis for the inhibition of eukaryotic zinc-dependent HDACs via mercaptoacetamide-based inhibitors

66 Dissous C, Grevelding C. Piggy-backing the concept of cancer drugs for schistosomiasis treatment: a tangible perspective? Trends Parasitol. 27(2), 59-66 (2011).

67 Schöffski P. Polo-Like Kinase (PLK) inhibitors in preclinical and early clinical development in oncology. Oncologist 14(6), 559-570 (2009).

68 Long T, Cailliau K, Beckmann S et al. Schistosoma mansoni Polo-like kinase 1: a mitotic kinase with key functions in parasite reproduction. Int. J. Parasitol. 40(9), 1075-1086 (2010).

69 Mcdermott J, Jimeno A. Belinostat for the treatment of peripheral T-cell lymphomas. Drugs Today 50(5), 337-345 (2014).

70 Kannan S, Melesina J, Hauser A et al. Discovery of inhibitors of Schistosoma mansoni HDAC8 by combining homology modeling, virtual screening, and in vitro validation. J. Chem. Inf. Model. 54(10), 3005-3019 (2014).

- Describes a virtual screening strategy that led to discovery of drug-leads exhibiting selective inhibition toward schistosome HDAC 8

71 Millard C, Watson P, Celardo I et al. Class I HDACs share a common mechanism of regulation by inositol phosphates. Mol. Cell 51(1), 57-67 (2013).

72 Watson P, Fairall L, Santos G, Schwabe J. Structure of HDAC3 bound to co-repressor and inositol tetraphosphate. Nature 481(7381), 335-340 (2012).

73 Lauffer B, Mintzer R, Fong R et al. Histone deacetylase (HDAC) inhibitor kinetic rate constants correlate with cellular histone acetylation but not transcription and cell viability. J. Biol. Chem. 288(37), 26926-26943 (2013).

74 Bottomley M, Lo Surdo P, Di Giovine P et al. Structural and functional analysis of the human HDAC4 catalytic domain reveals a regulatory structural zinc-binding domain. J. Biol. Chem. 283(39), 26694-26704 (2008).

75 Schuetz A, Min J, Allali-Hassani A et al. Human HDAC7 harbors a class IIa histone deacetylase-specific zinc binding motif and cryptic deacetylase activity. J. Biol. Chem. 283(17), 11355-11363 (2008).
76 Tang G, Wong J, Zhang W et al. Identification of a novel aminotetralin class of HDAC6 and HDAC8 selective inhibitors. J. Med. Chem. 57(19), 8026-8034 (2014).

77 Balasubramanian S, Ramos J, Luo W, Sirisawad M, Verner E, Buggy Jj. A novel histone deacetylase 8 (HDAC8)-specific inhibitor PCI-34051 induces apoptosis in T-cell lymphomas. Leukemia 22(5), 1026-1034 (2008).

78 Suzuki T, Muto N, Bando M et al. Design, synthesis, and biological activity of NCC149 derivatives as histone deacetylase 8-selective inhibitors. ChemMedChem 9(3), 657-664 (2014).

$79 \mathrm{Hu}$ J, Van Den Steen P, Sang Q, Opdenakker G. Matrix metalloproteinase inhibitors as therapy for inflammatory and vascular diseases. Nat. Rev. Drug Dis. 6(6), 480-498 (2007).

80 Sung Y, Lee T, Yoon $\mathrm{H}$ et al. Mercaptoacetamide-based class II HDAC inhibitor lowers $A \beta$ levels and improves learning and memory in a mouse model of Alzheimer's disease. Exp. Neurol. 239, 192-201 (2013).

81 Cole K, Dowling D, Boone M, Phillips A, Christianson D. Structural basis of the antiproliferative activity of largazole, a depsipeptide inhibitor of the histone deacetylases. J. Am. Chem. Soc. 133(32), 12474-12477 (2012).

82 Singh R, Suzuki T, Mandal T et al. Thermodynamics of binding of structurally similar ligands to histone deacetylase 8 sheds light on challenges in the rational design of potent and isozyme-selective inhibitors of the enzyme. Biochemistry 53(48), 7445-7458 (2014)

83 Somoza J, Skene R, Katz B et al. Structural snapshots of human HDAC8 provide insights into the class I histone deacetylases. Structure 12(7), 1325-1334 (2004).

84 Kunze M, Wright D, Werbeck N, Kirkpatrick J, Coveney P, Hansen D. Loop interactions and dynamics tune the enzymatic activity of the human histone deacetylase 8. J. Am. Chem. Soc. 135(47), 17862-17868 (2013).

85 Vennervald B, Polman K. Helminths and malignancy. Parasite Immunol. 31(11), 686-696 (2009).

86 Tsai I, Zarowiecki M, Holroyd $\mathrm{N}$ et al. The genomes of four tapeworm species reveal adaptations to parasitism. Nature 496(7443), 57-63 (2013).

87 Lane A, Chabner B. Histone deacetylase inhibitors in cancer therapy. J. Clin. Oncol. 10(27), 5459-5468 (2009).

88 Abdulla M, Ruelas D, Wolff B et al. Drug discovery for schistosomiasis: hit and lead compounds identified in a library of known drugs by medium-throughput phenotypic screening. PLoS Negl. Trop. Dis. 3(7), e478 (2009). 